\title{
Monitoring Methods of Crack Behavior in Hydraulic Concrete Structure Based on Crack Mouth Opening Displacement (CMOD)
}

\author{
Zhong-Yan Huo*, Guang-Xuan Qian, and Dong-Jian Zheng \\ ${ }^{1}$ College of Port and Waterway Engineering, University, Zhejiang Ocean University, Zhoushan, Zhejiang, 316000, \\ China \\ ${ }^{2}$ Changjiang River Scientific Research Institute Engineering Safety And Disaster Prevention Scientific, Wuhan, 430010 \\ ${ }^{3}$ Environmental Health Management Office of Dinghai District, Zhoushan, Zhejiang,316000,China
}

\begin{abstract}
For the difficulty of applying classical fracture criteria to the actual hydraulic engineering and silulating the process of cracking by conditional FEM, in this paper, a new method of analyzing and monitoring crack behavior in hydraulic structures under the effect of Hydro-Mechanical (HM) interaction is studied by using the XFEM, in which crack mouth opening displacement (CMOD) is adopted as monitoring index. The core innovation done in this study is that a method of determining macro crack tip is proposed based on cohesive force for the first time, and the critical value of CTOD is investigated and proved to be a material parameter. it is verified by comparing with the concrete tests and numerical calculations of former related literatures. All of the above shows that the present monitoring method based on CMOD provides a practical way to simulate cracking in hydraulic concrete structures.
\end{abstract}

Keywords: CMOD, cohesive crack, hydraulic concrete, monitoring method, XFEM.

\section{INTRODUCTION}

For some external or internal factors, certain parts of a hydraulic concrete structure may suffer a tension greater than the maximum tensile strength that the material can provide, which leads to the cracking of the structure during its construction or operation period. Obviously, the existence of cracks is seriously undermined the integrity of a structure, having a significant impact on the structure itself and its morphology, and is not conducive to operate the structure safely. The influence varies from the location, length and depth of the cracks. Therefore, monitoring and analyzing the deformation of cracks is one of the important contents for safety evaluation of a hydraulic concrete structure.

A lot of studies have done on the theory of formation, evolution and monitoring of cracks in hydraulic concrete stuctures. Currently, the most popular criteria determining whether cracks will extend or unstable are the maximum principal stress criterion, the crack-tip stress intensity factor criterion, the crack-tip opening displacement $\left(\mathrm{CTOD}_{\mathrm{c}}\right)$ criterion and so on. However, there is a difficulty to apply these theoretically feasible criteria to the actual engineering. Considering that some joint meter are often laid in cracks to measure the crack mouth opening displacement (CMOD) of hydraulic concrete structures, but there isn't a unified standard for applying CMOD as the parameter of crack

*Address correspondence to this author at Changzhou Road, Zhoushan, Zhejiang, China. Postcard: 316000; Tel: + 86 188570931601;

Fax: +86 05802550401; E-mail: hhuhzy@ yahoo.com extension criterion. Nevertheless, criteria for crack extention based on CMOD can be built through the relationships between CMOD and other variables such as CTOD. It is not only close to actual engineering, but also easier to be mastered by technical staff and management personnel. However, as the crack size changes during the operation of the structure, it is necessary to mesh and remesh the discontinuity surfaces, thus increasing the computational costs and projection errors associated with conventional finite element methods. In recent years, the development of the numerical analysis for solving discrete mechanics, such as XFEM, facilitates the building of the deformation monitoring methods for those with non-stable cracks.

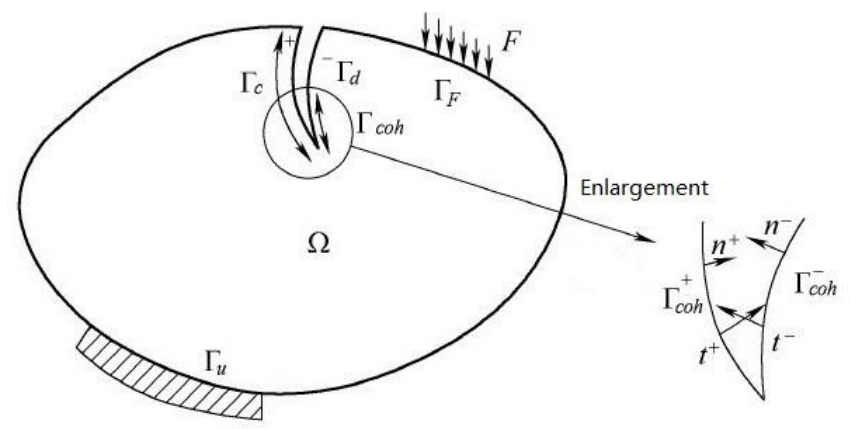

Fig. (1). Enlargement of viscous region.

The extended finite element method (XFEM), first introduced by U.S. Northwestern University Study Group 
headed by Prof. Belytschko [1], provides a convenient and effective way for problems with discontinuities. It models the discontinuity in a displacement field along the crack path, wherever this path may be located without respecting to the mesh. This flexibility enables the method to simulate crack growth without remeshing. XFEM has been developed rapidly and applied widely in only a few years [2-14]. Since the crack formation and expansion of actual concrete structures can be described as the process from ductile to brittle, thus the softening behavior of concrete is significant. Currently, there are mainly two kinds of fracture models can be used to silmulate concrete cracking process, that is separated crack model and smeared crack model. As the hydrualic concrete structure with cracks is always operated under the complex environment of Hydro-Mechanical interaction which couldn't be ignored; and the separated crack models are able to model the impact of water pressure within cracks. Thus the cohesive crack model (CCM), which is the most popular one of the separated crack models and is proposed firstly by professor hillerborg according to the cracking characteristics of concrete, should be the suitable fracture model. In fact, the cohesive crack model has been introduced to XFEM and then used to model the concrete crack propagation by many experts at home or abroad. For example, Moes, $\mathrm{N}$ and T.Belytschko [9] introduce CCM to XFEM. The cohesive zone growth is governed by requiring the SIFs at the cohesive zone tip to vanish, and the full amount of the displacement is taken as the variable, and then the effectiveness is demonstrated by simulations of cohesive crack growth in concrete. Unger, J F. et al [10] use the CCM to investigate that different criterion for predicting the direction of the extension of a cohesive crack in the context of XFEM. Asferg, J. L. et al. do the similar work as Unger, J. F, but their scheme is implemented and demonstrated to be reliable both for the 3-node constant strain triangle and the 6node linear strain triangle. Domestic scholars Fang Xiujun et al. develop a virtual node method which embeds the XFEM and CCM into ABAQUS [12], and build a coupling model for interaction between fissure water and cracking in concrete [13]. Regardless of the constitutive model of cohesive zone, the above studies have a common ground that only elements cut by the crack is enriched; in that case, crack tip could only be in the boundary of the elements, and some problems such as convergence problems and the fitted curve may not be satisfactory. Therefore, in this study, the CCM based on the XFEM considering crack-tip enriched is deduced firstly, which could make up the deficiency of these methods and improve the accuracy, and the crack-tip enriched function is taken according to an asymptotic analysis $[15,16]$ of the mechanical fields in a cohesive zone for very large structure and proved to be reasonable, and then the method is used to build the interaction model between fissure water and cracking in concrete. Secondly, the above model is used to analyze the relationships between the CMOD and other variables such as CTOD during the whole process of crack extension, and then to build the CMOD based creatia of crack extention. Finally, from all the above, the monitoring method of crack extension in hydraulic concrete structure, based on CMOD, is established from crack initiation to unstable expansion.

\section{MATERIALS AND METHODOLOGY}

\subsection{The Extended Finite Element Discretization}

The basic idea of XFEM is to make the crack path been located without any respect to the mesh by using some additional function to improve classical FEM. XFEM models the discontinuity in a displacement field along the crack path by incorporating some local enrichment functions into the classical finite element approximation. The displacement field approximation can be expressed as [3]:

$\boldsymbol{u}_{T}=N \boldsymbol{u}+N(H-H(\boldsymbol{x})) \boldsymbol{a}+N(\phi-\phi(\boldsymbol{x})) \boldsymbol{b}$

Where, $N$ is the array of shape functions; $\boldsymbol{u}, \boldsymbol{a}$ and $\boldsymbol{b}$ represent the vectors of displacement and enriched variables related to nodes. $H$ involves the Heaviside function, and $\phi$ is used to model the displacement field around the tip of the discontinuity. According to the asymptotic analysis $[15,16]$ of the mechanical fields in a cohesive zone for very large structure, $\phi$ is taken as:

$\phi=\left[r \sin \frac{\theta}{2}\right]$

Where $(r, \theta)$ is the local polar coordinates at the tip.

\subsection{Crack Extension of Hydraulic Concrete Structure}

Considering that the crack formation and expansion of actual concrete dam can be described as the process from ductile to brittle, the cohesive crack model is adopted as the fracture model. As shown in Fig (2), computational domain is $\Omega$, including the crack region $\Gamma_{c} . \Gamma_{c}$ is divided into fracture zone $\Gamma_{d}$ (displacement independent and no stress passed on between the two sides) and cohesive zone $\Gamma_{c o h}$ (displacement discontinuity but stress transferred between two sides). Displacement field of domain $\Omega$ :

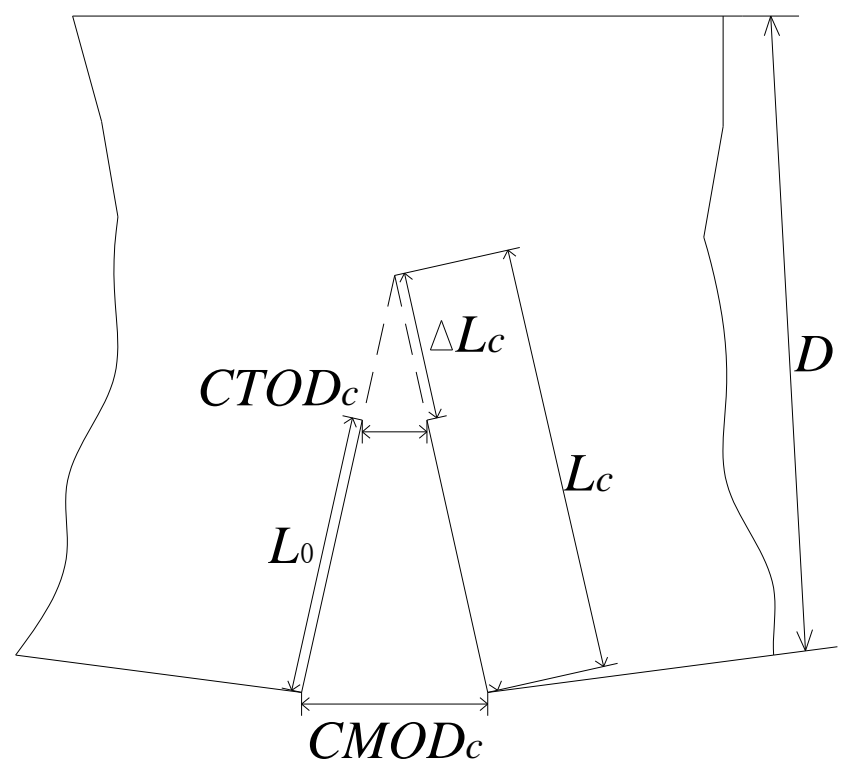

Fig. (2). Schematic configuration of crack initiation. 


\subsubsection{Cohesive Crack Extension}

The vector of displacement $\boldsymbol{u}_{T}$ complys with the principle of kinematics, thus weak form of the problem is given as follows:

$\boldsymbol{u} \in \mu=\{\boldsymbol{v} \in v: v=0$ on $\Omega\}$

In space $v$, discontinuity on the bountry $\Gamma_{c}$ is allowed, then the equation of virtual work without the effect of physical strength is:

$\int_{\Omega} \nabla v: \sigma d \Omega=\int_{\Gamma_{F}} v: \bar{t} d \Gamma$

Where $\bar{t}$ represents the external force acting on the boundary. According to the principles of virtual work, taking the variational on the continuous component and discontinuous component of the displacement mode respectively.

$\int_{\Omega} \delta \boldsymbol{u}^{\mathrm{T}} B^{\mathrm{T}} \boldsymbol{\sigma} d \Omega=\int_{\Gamma_{\digamma}} \delta \boldsymbol{u}^{\mathrm{T}} N^{\mathrm{T}} \overline{\boldsymbol{t}} d \Gamma$

$\int_{\Omega} \delta \boldsymbol{a}^{\mathrm{T}} H B^{\mathrm{T}} \boldsymbol{\sigma} d \Omega+\delta \Pi_{\text {crack }}=0$

$\int_{\Omega} \delta \boldsymbol{b}^{\mathrm{T}} \phi B^{\mathrm{T}} \boldsymbol{\sigma} d \Omega+\delta \Pi_{\text {crack }}=0$

In the above equation, " $\delta \prod_{\text {crack }}$ " is the virtual work done by cohesive force:

$\delta \Pi_{\text {crack }}=\int_{\Gamma_{\text {cooh }}} \delta \boldsymbol{w}_{l}^{\mathrm{T}} \boldsymbol{t s} d \Gamma$

Where $S$ is the area of unilateral crack per unit length as the crack expending; and $t$ represents the cohesive force within the crack, which is the function of CMOD. If $w(v)$ denotes CMOD, then there exists a equation under the global coordinate system as follows: $\boldsymbol{w}_{x-y}=\left(N_{c o h}^{+}-N_{c o h}^{-}\right) \boldsymbol{v}$, which could be converted to $\boldsymbol{w}_{l}$ under the local coordinate by the transformation matrix $\boldsymbol{M}$, that is, $\boldsymbol{w}_{l}=\boldsymbol{M} \boldsymbol{w}_{x-y} \cdot N_{c o h}^{+}$and $N_{c o h}^{-}$ are the shape functions on the " $\Gamma_{c o h}^{+}$" and " $\Gamma_{\text {coh }}^{-}$" side of the crack repectively. $N_{c o h}^{+}=N, N_{c o h}^{-}=-N$ if the cohesive zone is in split elements. Both $N_{c o h}^{+}$and $N_{c o h}^{-}$are expressed as $N \phi$, if the cohesive zone is in tip elements, and they are equal in magnitude but have opposite sign. If the incremental form of $\boldsymbol{t}$ is expressed as $\dot{\boldsymbol{t}}$, then:

$\dot{\boldsymbol{t}}\left(\boldsymbol{w}_{l}\right)=\frac{\partial \boldsymbol{t}}{\partial \boldsymbol{w}_{l}} \dot{\boldsymbol{w}}_{l}=T \dot{\boldsymbol{w}}_{l}=T M \dot{\boldsymbol{w}}_{x-y}$

Where $T$ is the material matrix of cracks, and index softening constitutive[17] is adopted as $T$ in this study.

The incremental form of stress is expressed as follows:

$\dot{\boldsymbol{\sigma}}=D \dot{\boldsymbol{\varepsilon}}=D\left(B_{u} \dot{\boldsymbol{u}}+B_{a} \dot{\boldsymbol{a}}+B_{b} \dot{\boldsymbol{b}}\right)$

Where $B_{u}, B_{a}$ and $B_{b}$ represent strain matrices of conventional, $H$ function enriched and crack-tip enriched degrees of freedom (DOFs) respectively.
The discrete form of governing equation could be obtained as follows by bringing equation (6) (8) into the equation (5):

$\left[\begin{array}{cc}K_{u u} & K_{u u^{\prime}} \\ K_{u^{\prime} u} & K_{u^{\prime} u^{\prime}}+K_{c o h}\end{array}\right]\left[\begin{array}{c}\dot{\boldsymbol{u}} \\ \dot{\boldsymbol{u}}^{\prime}\end{array}\right]=\left[\begin{array}{c}\boldsymbol{f}^{\mathrm{ext}} \\ 0\end{array}\right]-\left[\begin{array}{c}\boldsymbol{f}_{u}^{\mathrm{int}} \\ \boldsymbol{f}_{u^{\prime}}^{\mathrm{int}}\end{array}\right]$

Where:

$K_{u u}=\int_{\Omega} B_{u}^{T} D B_{u} d \Omega$

$K_{c o h}=\int_{\Gamma_{c o h}}\left(N_{c o h}^{+}-N_{c o h}^{-}\right)^{T} M^{T} T M\left(N_{c o h}^{+}-N_{c o h}^{-}\right) d \Gamma$

$\boldsymbol{f}_{u}^{\mathrm{int}}=\int_{\Gamma_{F}} B_{u}{ }^{T} \dot{\boldsymbol{\sigma}} d \Gamma=\int_{\Gamma_{F}} B_{u}{ }^{T} D B_{u} \dot{\boldsymbol{u}} d \Gamma$

$\boldsymbol{f}_{u^{\prime}}^{\mathrm{int}}=\int_{\Gamma_{c o h}} B_{u^{\prime}}{ }^{T} D B_{u} \dot{\boldsymbol{u}} d \Gamma+\int_{\Gamma_{c o h}}\left(N_{c o h}^{+}-N_{c o h}^{-}\right)^{T} M^{T} \overline{\boldsymbol{t}} d \Gamma$

In the above, $u$ and $u^{\prime}$ represent conventional and enriched part repectively; $\boldsymbol{f}_{u}^{\text {int }}$ and $\boldsymbol{f}_{u^{\prime}}^{\text {int }}$ are the internal forces of nodes. Therefore, the right hand side of the equation (9) means unbalanced force of node. $\boldsymbol{f}_{u^{\prime}}^{\text {int }}=0$, since the tensile stress within the crack is continuous. If the cohesive zone is in split elements, then:

$$
\begin{array}{r}
K_{u u^{\prime}}=K_{u^{\prime} u}=\int_{\Omega} B_{u}^{T} D B_{u}(H-H(x)) d \Omega \\
=\int_{\Omega}(H-H(x)) B_{u}{ }^{T} D B_{u} d \Omega \\
K_{u^{\prime} u^{\prime}}=\int_{\Omega} B_{a}^{T} D B_{a} d \Omega
\end{array}
$$

If the cohesive zone is in tip elements, then:

$K_{u u^{\prime}}=K_{u^{\prime} u}=\int_{\Omega} B_{b}^{T} D B_{u} d \Omega$

$K_{u^{\prime} u^{\prime}}=\int_{\Omega} B_{b}^{T} D B_{b} d \Omega$

Where, $\boldsymbol{u}, \boldsymbol{a}$ and $\boldsymbol{b}$ are respectively the variables related to nodes enriched DOFs of the conventional, $H$ function enriched and crack-tip; $B_{i}^{r}(r=u, a, b)$ are the corresponding strain matrices varied by $r$ and $i$, where $i$ is the node number of elements.

\subsubsection{Water Pressure Distribution Within Cracks}

In fact, the force acting on the cohesive zone of concrete cracks can be considered as superimposed effect of the cohesive force and water pressure, which could be simplified as follows according to Bruhwiler [17]:

$$
p_{\text {coh }} / p_{\max }=3\left(\frac{w}{\kappa}\right)^{2}-2\left(\frac{w}{\kappa}\right)^{3}
$$

Where $p_{\text {coh }}$ represents the water pressure within the cohesive zone of cracks, $w$ is on behalf of the fictitious opening displacement of cracks, and $p_{\max }$ means the maximum water pressure within cracks. $\kappa$ is the parameter related to the force within cracks, which is taken as the maximum of CMOD in this paper. 
As the entering of water flow, the force acting on the macroscopic zone of concrete crack is mainly the hydrostatic pressure which is perpendicular to the crack surface:

$p_{\text {静 }}=\gamma_{w}(H-z)$

Where $H$ and $z$ are respectively the water heads of a node and certain position.

\subsubsection{Incremental Equations of Crack Propagation}

Constitutive relationship of the cohesive crack is finally reflected in the additional stiffness matrix of overall equation, while water pressure of the macroscopic crack is finally reflected in the second term of load array, which is in the right side of the equation. Governing equation is still deduced by the incremental iterative method, and its discrete form can be expressed as follows:

$$
\left[\begin{array}{cc}
K_{u и} & K_{u u^{\prime}} \\
K_{u^{\prime} u} & K_{u^{\prime} u^{\prime}}+K_{c o h}
\end{array}\right]\left[\begin{array}{c}
\dot{\boldsymbol{u}} \\
\dot{\boldsymbol{u}}
\end{array}\right]=\left[\begin{array}{c}
\dot{\boldsymbol{f}}_{\text {ext }} \\
0
\end{array}\right]+\left[\begin{array}{c}
0 \\
\dot{\boldsymbol{P}}_{u^{\prime}}^{\text {ext }}
\end{array}\right]-\left[\begin{array}{c}
\boldsymbol{f}_{u}^{\text {int }} \\
\boldsymbol{f}_{u^{\prime}}^{\text {int }}
\end{array}\right]
$$

Where:

$\boldsymbol{f}_{u^{\prime}}^{\mathrm{int}}=\int_{\Gamma_{c o h}} B_{u^{\prime}}{ }^{T} D B_{u} \dot{\boldsymbol{u}} d \Gamma+\int_{\Gamma_{c o h}}\left(N_{c o h}^{+}-N_{c o h}^{-}\right)^{T} M^{T} \dot{\boldsymbol{P}}_{c o h} d \Gamma ; \dot{\boldsymbol{P}}_{c o h}$ is the incremental force within the cohesive zone of the crack, and it is superimposed by the cohesive force and water pressure. $\dot{\boldsymbol{P}}_{u^{\prime}}^{\text {ext }}$ means the incremental form of water pressure within the crack.

\subsection{Monitoring Index of Crack Deformation}

Generally speaking, $\mathrm{CTOD}_{\mathrm{c}}$ (the critical value of CTOD) is the property parameter of concrete material, while $\mathrm{CMOD}_{\mathrm{c}}$ (the critical value of CMOD) is not only affected by the material property, but also related to the layout of joint meters, the depth and cohesive zone of cracks. Therefore, monitoring methods based on CMOD should be built by studying the relationship between CTOD and CMOD. At present, it is when the crack unstable that the relationship between CTOD and CMOD is better studied by cracking test of concrete [18]; such as literature [19], which proved that it is necessary to consider the effect of cohesive length of concrete crack and studied the relationship by the fracture test of wedged compact tension as follows(Shown in Fig. 2):

$C T O D_{c}=\frac{\Delta L_{c}}{L_{c}} C M O D_{c}=\frac{\Delta L_{c}}{\Delta L_{c}+L_{0}} C M O D_{c}$

Where $\mathrm{L}_{0}$ and $\mathrm{D}$ are respectively the initial crack length and the height of specimen; $\mathrm{L}_{c}$ is the critical crack length; $\Delta \mathrm{L}_{c}$ means the length of virtual crack, that is, the distance between the virtual and macroscopic crack-tip, which are respectively the forefront and end point of the cohesive zone. meantime, the macroscopic crack-tip is the first point where cohesive force is zero.
However, the CTOD usually measured by the fracture test of concrete is its initial value at a fixed position. In fact, both the virtual and macroscopic crack-tip are forward dynamically during the cracking process. The positions of the crack-tips change while the crack is extending, and they aren't at the initial positions since crack initiation to the time the structure destroys of being instability. Therefore, the method combining cohesive crack model with XFEM, which is different from the concrete fracture tests used by previous investigators, is adopted to track the expansion of crack-tip and to analyze the relationships between the CMOD and the opening displacement of macroscopic crack-tip and the length of cohesive zone or other variables during the whole process from crack initiation, stable extension to the critical state of unstable extension. Finally, the monitoring methods of crack behavior based on CMOD are studied.

As shown in Fig. (3), concrete plate with a horiontal seam is used as the case model. Its length and width are respectively $8 \mathrm{~m}$ and $6 \mathrm{~m}$. The initial length of the seam is $0.2 \mathrm{~m}$, and the seam is $0.9 \mathrm{~m}$ away from the bottom of the plate. The area which is upper and lower the position of the seam within the range of $0.3 \mathrm{~m}$ is assumed as a weak zone, as shown in Fig. (3) with the shaded.

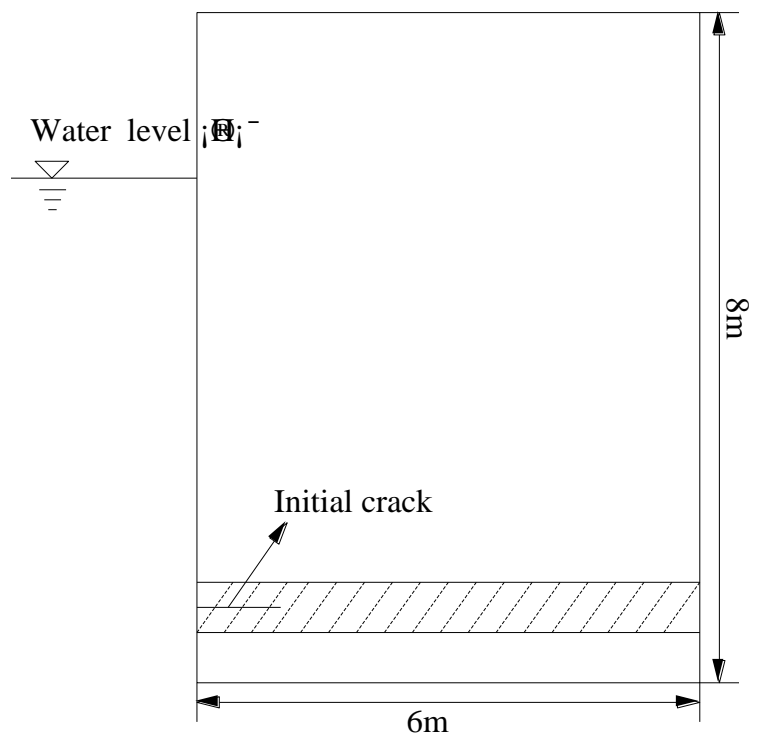

Fig. (3). Concrete plate.

The CCM are suitable for considering the impact of water pressure within the crack, so the model is used to analyze the extension of crack. Maximum tensile stress criterion is adopted, and index softening constitutive is used in the cohesive zone of the crack. Material properties of the concrete are shown in Table $\mathbf{1}$. It is assumed that the plate is destroyed by tensile.

General procedure: The extension of the crack is simulated by the method proposed in section 2.1. Each step

Table 1. Material parameters of the concrete.

\begin{tabular}{|c|c|c|c|c|}
\hline $\boldsymbol{\rho ~ k g} / \mathbf{m}^{\mathbf{3}}$ & $\mathbf{E ~ G P a}$ & $\boldsymbol{v}$ & Tensile strength $\mathbf{f}_{\mathbf{t}}(\mathbf{M p a})$ & Fracture energyG $\mathbf{f}(\mathbf{N} / \mathbf{m})$ \\
\hline \hline 2350 & $2 \times 10^{10}$ & 0.167 & $1.78(0.7)$ & $120(30)$ \\
\hline
\end{tabular}

Notes: The values of parameters in parentheses mean the corresponding values near the weak zone. 
the crack extends, its virtual crack-tip will move forwad one step, and cohesive force within the crack will be redistributed. Therefore, the iterative method is proposed to determine the length of cohesive zone and the position of macroscopic crack-tip, and could be expressed as follows: (1) Giving initial length of the crack (including its cohesive zone) firstly; and then solving the opening diplacement of each point on the crack surface. It is noteworthy that the cohesive force needn't calculating until the crack initiation.(2) Iterating through the energy convergence criterion since the $2^{\text {nd }}$ iterative step, and then the cohesive forces and the actual opening displacements of each point within the crack could be calculated based on its corresponding opening displacements. (3) Index softening constitutive which means that the cohesive force exponentially decreases with the increasing of the CMOD, is adopted in this study. As the value of the cohesive force is less than a certain value, the effect of CMOD on the cohesive force could be ignored according to the characteristics of the exponential function. Therefore, a threshold of the normal cohesive force, which is corresponding to the $\mathrm{CTOD}_{\mathrm{c}}[20]$, could be set to determine whether the value of cohesive force is zero. If the value of cohesive force at a point within the crack is less than this threshold, then the point is considered to be in the macroscopic zone of the crack. Through the cost calculator, this threshold and its corresponding value of $\mathrm{CTOD}_{\mathrm{c}}$ are respectively taken as $6.5 \times 10^{2} \mathrm{~N}$ and $5.25 \times 10^{-4} \mathrm{~mm}$. (4) Through interpolating with the known integral points, the coordinate of the threshold point, also called macroscopic crack-tip, could be culculated. The distance between the virtual crack-tip and macroscopic crack-tip is the length of cohesive zone.

For the reason that the effect of the step length adopted in numerical simulation of the horizontal crack is significant, if the step lenth is taken too large, then the true ultimate bearing capacity may not be caught; while the value is taken too small, it will increse the computational efforts. Through the cost calculator, the step length is taken as $0.015 \mathrm{~m}$.

\section{RESULTS}

The relationship-curves between the CMOD and CTOD in the first three steps of the crack extension are given in Fig. (4).
As shown in Fig. (4), the relationships between CMOD and CTOD in the first three steps of the crack extension are consistent with each other. The process of new crack-tip extends in each step is similar to that in the first step. A slowly rising and non-linear phase appear firstly, and then the series of relationship-curves tend to be horizontal lines. Each time the stress near new crack-tip meets the fracture criterion, the $\mathrm{CTOD}_{\mathrm{c}}$ remains unchanged. It shows that "CTOD ${ }_{\mathrm{c}}$ " is a material parameter and independent of the size of structures. However, this conclusion is inconsistent with that of literature [19], in which "CTOD ${ }_{\mathrm{c}}$ " is dependent of the size of structures. The cause could be analyzed as follows. In concrete fracture tests, the position of macroscopic crack-tip is predetermined and fixed, and the opening displacement of this fixed crack-tip is measured as CTOD, which is still the value of initial crack-tip wherever the crack extends.

Meanwhile, in order to analyze the impact of the cohesive crack zone, bringing the calculation results of $\mathrm{CMOD}_{\mathrm{c}}, \Delta \mathrm{L}_{\mathrm{c}}, \mathrm{L}_{\mathrm{c}}$ and other parameters into the equation (21). Consequently, the results of $\mathrm{CMOD}_{\mathrm{c}}$ (average of each extension step) considering the impact of cohesive zone and that without considering the impact are repectively $5.2304 \times 10^{-2} \mathrm{~mm}$ and $5.0639 \times 10^{-2} \mathrm{~mm}$. Comparing with $5.2518 \times 10^{-2} \mathrm{~mm}$, which is the value of $\mathrm{CMOD}_{\mathrm{c}}$ obtained by the method proposed in section 2.1, the relative errors of the former two are repectively $0.41 \%$ and $3.58 \%$. All the above results indicate that the value of $\mathrm{CMOD}_{\mathrm{c}}$ calculated without considering the cohesive effect is relatively small.

To better verify the reliability of the method proposed in section 2.1, it is adopted to analyze the relationship-curve between CMOD and CTOD during the whole process of crack extension, in which the crack-tip is fixed. As shown in Fig. (5), the curve could mainly be divided into three sections: (1) Rising phase before the crack initiation. (2) After initiation, the crack extends stablely with the increase of water level; it destroys of being instability whlie water level increase to the ultimate load, and the values of CMOD and CTOD at this time are respectively their critical values $\mathrm{CMOD}_{\mathrm{c}}$ and $\mathrm{CTOD}_{\mathrm{c}}$. (3) Unstable extension phase of crack. In this period, the crack will continue extending at the same water level or an even lower level, that is, the curve has a downward trend and the bearing capacity of structure weakened. Threrefore, when the crack-tip position is fixed, the conclusion obtained by the proposed method is

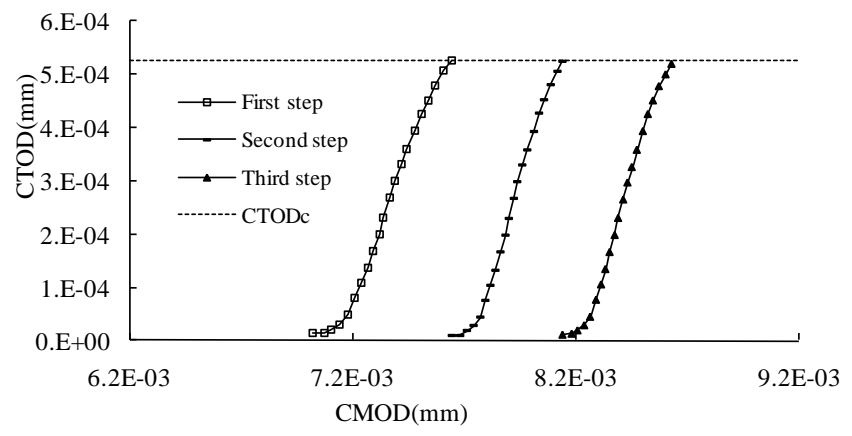

Fig. (4). Relationships between CMOD and CTOD at the first three steps of crack extension. 


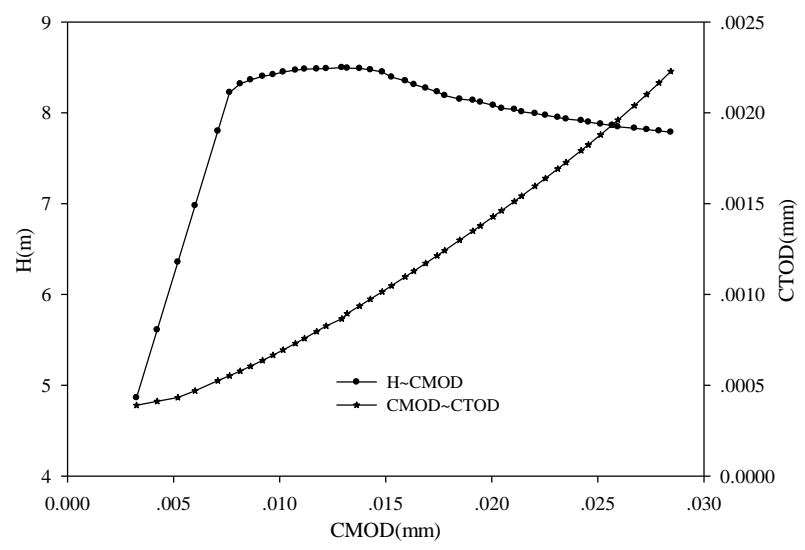

Fig. (5). Relationship between CMOD and CTOD

consisitent with that of literature [19], and the method proposed in this paper is reliable.

\subsection{Monitoring Methods of Crack Behavior based on CMOD}

Known from section 2.2, after initiation, the crack is firstly in a stable phase of expansion, and then extends progressively with the increase of the water load, correspondingly, the crack-tip moves foward until the crack destroys of being instability. By simulating the process of crack expension according to the method given in section $\mathbf{2 . 1}$ and 2.2, the values of $\mathrm{CMOD}_{\text {inic }}, \mathrm{CMOD}_{\mathrm{ci}}$ and $\mathrm{CMOD}_{\mathrm{unc}}$ could be obtained and then be used to built the monitoring methods of crack behavior based on CMOD. The three kinds of CMOD mentioned above are respectively the thresholds of its initial value, the value of its $i^{\text {th }}$ sable extension and its value of being instability.

\subsubsection{Monitoring Method of Crack Initiation}

As cracks start to expand, the value of $\mathrm{CMOD}_{\text {inic }}$ could be calculated by equation (22) as follows:

$C M O D_{\text {inic }}=\left(\Delta L_{c 1}+L_{0}\right) / \Delta L_{c 1} C T O D_{c}$

Where $\Delta \mathrm{L}_{\mathrm{c} 1}$ is the length of virtual crack in the first step of crack extension. Once $\mathrm{CMOD}_{\text {inic }}$ is obtained, the monitoring method for crack initiation could be built as follows:

If $C M O D<C M O D_{\text {inic }}$, then the crack is in a stable state;

If $C M O D=C M O D_{\text {inic }}$, then the crack is in the critical state before initiation;

If $C M O D>C M O D_{\text {inic }}$, then the crack is in the state of stable extension.

\subsubsection{Monitoring Method for the Stable Stage of Crack Extension}

As the increase of water load and cracks start to expand, firstly cracks are in the state of stable extension, both the length of crack and the CMOD are gradually increasing. If the length of crack is $\mathrm{L}_{i-1}$ after the (i-1) ${ }^{\text {th }}$ stable extension, then the value of $\mathrm{CMOD}_{\mathrm{ci}}$ could be calculated by equation (23) as follows (Shown in Fig. 6):

$$
C M O D_{c i}=\left(\Delta L_{c i}+L_{i-1}\right) / \Delta L_{c i} C T O D_{c}
$$

Where $\Delta \mathrm{L}_{\mathrm{ci}}$ is the length of the virtual crack in the $\mathrm{i}^{\text {th }}$ step of crack extension, and could be obtained by the method given in section 2.1. and 2.2. Once $\mathrm{CMOD}_{\mathrm{ci}}$ is obtained, the monitoring method for the $\mathrm{i}^{\text {th }}$ stable extension of cracks could be built as follows:

If $C M O D<C M O D_{c i}$, then the crack is in a stable state without extending;

If $C M O D=C M O D_{c i}$, then the crack is in the critical state before the $\mathrm{i}^{\text {th }}$ stable extension;

If $C M O D>C M O D_{c i}$, then the crack is in the state of the $i^{\text {th }}$ stable extension.

\subsubsection{Monitoring Method for the Unstable Stage of Crack Extension}

As cracks extend since the stable state of extension until destorying of unstable extension. Then the monitoring method for the unstable extension of cracks could be built as follows:

If $C M O D<C M O D_{\text {unc }}$, then the crack is in the state of stable extension;

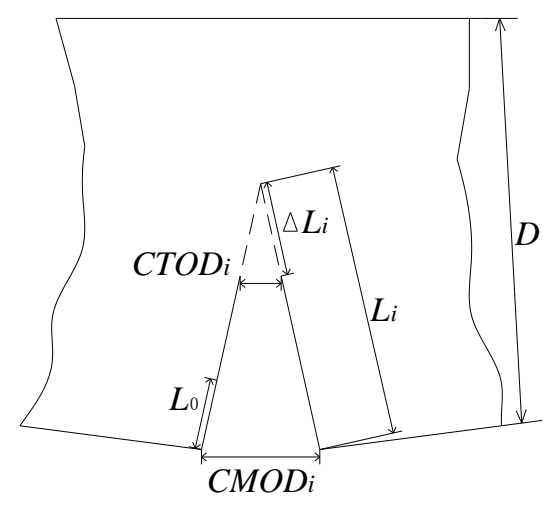

Fig. (6). Schematic configuration of crack opening at the step $i$. 
If $C M O D=C M O D_{u n c}$, then the crack is in the critical state before unstable extension;

If $C M O D>C M O D_{u n c}$, then the crack is destroyed of unstable extension.

Where $C M O D_{\text {unc }}$ could be calculated by the method given in section $\mathbf{2 . 1}$ and $\mathbf{2 . 2}$.

\section{CONCLUSION}

The security status of hydraulic concrete structure, which is under the effect of Hydro-Mechanical (HM) interaction, is directly affected by the mechanic behavior of cracks. In this paper, based on analysis of the properties of hydraulic concrete structure and with the cohesive crack model (CCM), the analysis and monitoring method of the security status of hydraulic concrete structure crack under the effect of HM interaction is studied by using the XFEM.

(1) Tip-element effect of the XFEM is included in solving the problem of interaction between fissure water and cracking in concrete. With the cohesive crack model, the incremental iterative method of the nonlinear equation group and corresponding discrete form are deduced and shown in detail.

(2) A method of determining macro crack tip is proposed based on cohesive force. The critical crack-tip opening displacement $\left(\mathrm{CTOD}_{\mathrm{c}}\right)$ of hydraulic structural cracks is investigated and proved to be a material parameter by the metod propose in this paper. The relationship between the crack mouth opening displacement(CMOD) and the cracktip opening displacement(CMOD) is obtained during the whole process of crack extension.

(3) A set of criteria related to crack initiation, stable expansion and unstable expansion are proposed, and then the safety monitoring method of crack extension in hydraulic concrete structure, based on CMOD, is established during the whole cracking process.

\section{CONFLICT OF INTEREST}

The authors confirm that this article content has no conflict of interest.

\section{ACKNOWLEGEMENTS}

The Special Fund Support of Natural Science Foundation of China(Grant Nos.51279052) is gratefully acknowledged.

\section{REFERENCES}

[1] T. Belytschko, and T. Black, "Elastic crack growth in finite elements with minimal remeshing," Int. J. Numer. Meth. Eng., vol. 45, no. 5, pp. 601-620, 1999.
[2] T. Belytschko, and D. Organ, "Element-free galerkin methods for dynamic fracture in concrete," Comput. Methods in Appl. Mech. Eng., vol. 187, no. 3, pp. 385-399, 2000.

[3] M. Stolarska, and D.L. Chopp, "Modelling crack growth by level sets in the extended finite element method," Int. J. Numer. Meth. Eng., vol. 51, no. 8, pp. 943-960, 2001.

[4] N. Sukumar, and J.H. Prevost, "Modeling quasi-static crack growth with the extended finite element method Part I: Computer implementation," Int. J. Solids and Struct., vol. 40, no. 26, pp. 7513-7537, 2003.

[5] T. Belytschko, and R. Gracie, "On XFEM applications to dislocations and interfaces," Int. J. Plasticity, vol. 23, no. 10, pp. 1721-1738, 2007.

[6] J. Dolbow, and I. Harari, "An efficient finite element method for embedded interface problems", Int. J. Numer Meth. Eng., vol. 78, no. 2, pp. 229-252, 2009.

[7] Y. J. Xu, and H. Yuan, "On damage accumulations in the cyclic cohesive zone model for XFEM analysis of mixed-mode fatigue crack growth", Comp. Mater. Sci., vol. 46, no. 3, pp. 579-585, 2009.

[8] J. Shi, and D. Chopp, "Abaqus implementation of extended finite element method using a level set representation for threedimensional fatigue crack growth and life predictions," Eng. Fract. Mech., vol. 77, no.14, pp. 2840-2863, 2010.

[9] N. Moes, and T. Belytschko, "Extended finite element method for cohesive crack growth", Eng. Fract. Mech., vol. 69, no. 7, pp. 813833, 2002.

[10] J. F. Unger, S. Eckardt, and C. Konke, "Modeling of cohesive crack growth in concrete structures with the extended finite element method". Comput. Methods in Appl. Mech. Eng., vol. 196, no. 41, pp. 4087-4100, 2007.

[11] J. L. Asferg, P. N. Poulsen, and L. O. Nielsen, "A direct XFEM formulation for modeling of cohesive crack growth in concrete", Comput. Concrete., vol. 4, no. 2, pp. 83-100, 2007.

[12] X. J. Fang, F. Jin, and J. T. Wang, "Cohesive crack model based on extended finite element method", Tsinghua. Sci. Technol., vol. 3 pp. 344-347, 2007. (In Chinese).

[13] X. J. Fang, and F. Jin, "Coupling model for interaction between fissure water and cracking in concrete", SHUILI XUEBAO, vol. 38 , no. 12 , pp. 1466-1474, 2007. (In Chinese).

[14] A. Hillerborg, M. Modeer, and P. E. Petersson, "Analysis of crack formation and crack growth in concrete by means of fracture mechanics and finite elements", Cement Concrete Res., vol. 6 , pp. 773-782, 1976.

[15] J. Planas, and M. Elices, "Asymptotic analysis of a cohesive crack_1_Theoretical background", Int. J. Fract., vol. 55, no. 2, pp. $1 \overline{53}-\overline{1} 77,1992$.

[16] J. Planas, and M. Elices, "Asymptotic analysis of a cohesive crack_2_Influence of the softening curve", Int. J. Fract., vol. 64, no. 3, pp. 221-237, 1993.

[17] E. Bruwiler, and V. E. Saouma, "Water fracture ineraction in concrete Part 2: Hydrostatic Pressure in Cracks", ACI. Mater. J., vol. 92, no. 4, pp. 383-390, 1995.

[18] Z. M. Wu, S. L. Xu, and J. L. Wang, "Fracture toughness and critical crack tip opening displacement of concrete based on fictitious crack model", J China Three Gorges Univ (Natural Sciences), vol. 24, no.1, pp. 29-34,2002. (In Chinese).

[19] S. L. Xu, X. F. Zhang, and D. Bu, "Relationship Between Crack Tip Opening Displacement(CTOD) and Crack Mouth Opening Displacement (CMOD) throughout Development of Concrete Crack", Eng. Mech., vol. 28, no. 5, pp. 64-70, 2011. (In Chinese).

[20] G. Alfano, S. Marfia, and E. Sacco, "A cohesive damage-friction interface model accounting for water pressure on crack propagation", Comput. Methods in Appl. Mech. Eng., vol. 196, pp. 192-209, 2006. 